\title{
CHAINS OF MAXIMUM LENGTH IN THE TAMARI LATTICE
}

\author{
SUSANNA FISHEL AND LUKE NELSON
}

(Communicated by Jim Haglund)

\begin{abstract}
The Tamari lattice $\mathcal{T}_{n}$ was originally defined on bracketings of a set of $n+1$ objects, with a cover relation based on the associativity rule in one direction. Although in several related lattices the number of maximal chains is known, quoting Knuth, "The enumeration of such paths in Tamari lattices remains mysterious."

The lengths of maximal chains vary over a great range. In this paper, we focus on the chains with maximum length in these lattices. We establish a bijection between the maximum length chains in the Tamari lattice and the set of standard shifted tableaux of staircase shape. We thus derive an explicit formula for the number of maximum length chains, using the Thrall formula for the number of shifted tableaux. We describe the relationship between chains of maximum length in the Tamari lattice and certain maximal chains in weak Bruhat order on the symmetric group, using standard Young tableaux. Additionally, recently Bergeron and Préville-Ratelle introduced a generalized Tamari lattice. Some of the results mentioned above carry over to their generalized Tamari lattice.
\end{abstract}

\section{INTRODUCTION}

The Tamari lattices $\left\{\mathcal{T}_{n}\right\}$ have been intensely studied since their introduction by Tamari in Tam62]. Yet the problem of finding a formula for the number of maximal chains, a classic problem for any family of posets, remains unsolved for these lattices. Here we present what we envision as first steps towards the enumeration of maximal chains. Although our two results are simple, they do not appear to have been noticed before. Our work suggests not only that the original problem is not intractable, but that its solution may have an interpretation in terms of representation theory.

This note is an offshoot of a long-standing project of the first author, along with Grojnowski, on the combinatorics of higher dimensional Catalan polynomials. Recently Bergeron and Préville-Ratelle [BP11], also working on higher dimensional Catalan polynomials, generalized the Tamari lattices to $m$-Tamari lattices. The original Tamari lattices are the case $m=1$. Some of the results in the first part of this note concern the generalized lattice, whereas the results in the second half are for the $m=1$ case.

The lengths of the maximal chains in the Tamari lattices vary over a great range. The longest chains in $\mathcal{T}_{n}$ have length $\left(\begin{array}{l}n \\ 2\end{array}\right)$ and the unique shortest has length $n-1$.

Received by the editors March 26, 2012 and, in revised form, October 17, 2012.

2010 Mathematics Subject Classification. Primary 05A15, 06A07; Secondary 05E05, 05A17.

Key words and phrases. Tamari lattice, maximal chains, shifted tableaux.

This work was partially supported by Simons Foundation Collaboration Grant No. 209806.

(C)2014 American Mathematical Society 3343

Reverts to public domain 28 years from publication 
Part of our strategy for finding the number of maximal chains is to focus on each length separately. Here, we determine the number of chains in the Tamari lattice $\mathcal{T}_{n}$ of maximum length. We do this using a simple bijection to shifted tableaux, then use Thrall's formula Knu73 to count those. We are currently investigating other lengths. Preliminary results, based on numerical evidence, are encouraging.

The Tamari lattice is both a quotient and a sublattice of the weak order on the symmetric group [BW97, Rea06]. The study of maximal chains in the weak order on $\mathcal{S}_{n}$ has proven to be extremely fruitful. The Stanley symmetric functions, balanced labelings, and dual equivalence arose from work on the weak order on $\mathcal{S}_{n}$ Sta84, EG87, Hai92. It was with this rich history in mind that we began our investigation of the maximal chains in the Tamari lattices. The work of Stanley and Edelman-Greene shows that the chains in weak order have a meaning in representation theory: they index a basis for an irreducible representation of the symmetric group. We hope for a similar interpretation here, and the appearance of shifted tableaux supports this. To this end, we begin the study of the relationship between the maximal chains in the weak order and the maximal chains in the Tamari. We seek to understand the interplay between the modified Robinson-Schensted algorithm of [EG87] and the Tamari order, via $c$-sorting.

The outline of this note follows. In Section 2 we review the definition of the Tamari lattice, using the $m$-Tamari generalization in [BP11. We do not discuss the relationship of this definition to the original definition in terms of bracketings Tam62. We describe how to assign a tableau to each maximal chain in the lattice in Section 3 It is this correspondence which allows us to enumerate the chains of maximum length. In Section 4 we relate the Edelman-Greene bijection between maximal chains in weak order on the symmetric group and standard Young tableaux of staircase shape [EG87] to our work. We characterize the maximal chains in $\mathcal{S}_{n}$ which are maximum length chains in $\mathcal{T}_{n}$ when we view $\mathcal{T}_{n}$ as an induced subposet of $\mathcal{S}_{n}$, in order to show that the Edelman-Greene bijection encodes the Tamari order in this case.

\section{The TAmari lattice AND its COVERING RELAtion}

A partially ordered set, or poset, is a lattice if every pair of elements has a least upper bound, the join, and a greatest lower bound, the meet. We consider the Tamari lattice here; the Tamari lattice of order $n$ is denoted $\mathcal{T}_{n}$. See Figure 1 for $\mathcal{T}_{3} . \mathcal{T}_{n}$ has several equivalent definitions. In [Tam62, the vertices are proper bracketings of $n+1$ symbols and the cover relation is given by the associative rule. [Knu73] describes $\mathcal{T}_{n}$ as a poset of forests on $n$ nodes, BW97] gives the definition in terms of the scope sequences of these forests, and BB09] phrases the definition in terms of Dyck paths. It is this last form of the definition that BP11] generalizes. We make a very slight change and give their generalized definition in terms of certain partitions, which are better for our purposes.

Definition 2.1. An $(m, n)$-Dyck partition $\lambda=\left(\lambda_{1}, \lambda_{2}, \ldots, \lambda_{n}\right)$ is an integer sequence such that

(1) $\lambda_{1} \geq \lambda_{2} \geq \ldots \geq \lambda_{n}=0$ and

(2) for each $i$, we have $\lambda_{i} \leq m(n-i)$. 
It is well-known ([DM47]) that there are the $m$-Catalan number $\frac{1}{n m+1}\left(\begin{array}{c}(m+1) n \\ n\end{array}\right)$ of these partitions. The $(m, n)$-Dyck partitions are the vertices for the $m$-Tamari lattice; the case $m=1$ is the original Tamari lattice.

Definition 2.2 ([BP11] $)$. Let $\lambda=\left(\lambda_{1}, \lambda_{2}, \ldots, \lambda_{n}\right)$ be an $(m, n)$-Dyck partition. For each $i$ between 1 and $n$, there is a unique $k=k(\lambda, i) \leq i$ such that

(1) $\lambda_{j}-\lambda_{i}<m(i-j)$ for $j=k, \ldots, i-1$ and

(2) either $k=1$ or $\lambda_{k-1}-\lambda_{i} \geq m(i-k+1)$.

Suppose $\lambda_{i}>\lambda_{i+1}$ and $k=k(\lambda, i)$. Set $\lambda \lessdot \mu$, where $\mu=\left(\lambda_{1}, \ldots, \lambda_{k-1}, \lambda_{k}-\right.$ $\left.1, \ldots, \lambda_{i}-1, \lambda_{i+1}, \ldots, \lambda_{n}\right)$. The $m$-Tamari lattice $\mathcal{T}_{n}{ }^{(m)}$ is the set of $(m, n)$-Dyck partitions, together with the transitive closure of this covering relation $\lambda \lessdot \mu$.

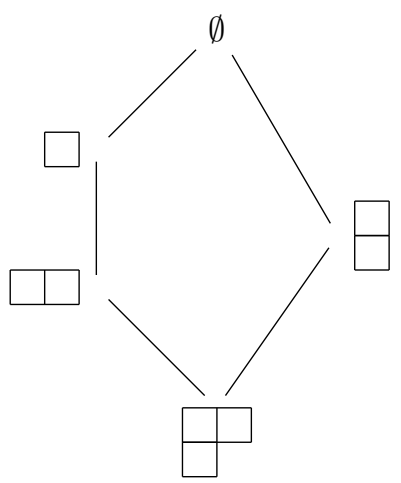

Figure 1. The Hasse diagram of the poset $\mathcal{T}_{3}$.

FT67 and also [HT72 showed that $\mathcal{T}_{n}=\mathcal{T}_{n}^{(1)}$ is a lattice. BFP11 showed that $\mathcal{T}_{n}{ }^{(m)}$ is a lattice and is in fact isomorphic to a sublattice of $\mathcal{T}_{n m}$. At the top of the lattice is the empty partition and at the bottom is the partition $((n-1) m, \ldots, m)$.

If a poset has an element $x$ with the property that $y \leq x$ for all $y$ in the poset, we denote that element by $\hat{1}$. Similarly, $\hat{0}$ is the element below all others if such an element exists. In a poset with $\hat{0}$ and $\hat{1}$, a maximal chain is a sequence of elements $\hat{0}=x_{0} \lessdot x_{1} \lessdot \ldots \lessdot x_{N-1} \lessdot x_{N}=\hat{1}$.

Let $\lambda=\left(\lambda_{1}, \ldots, \lambda_{r}\right)$ be an integer partition. Its Young diagram is an array of boxes, where there are $\lambda_{i}$ boxes in row $i$. The $(n, m)$-Dyck partitions are the partitions whose Young diagram fits inside the diagram of $(m(n-1), m(n-2), \ldots, m)$. A tableau of shape $\lambda$ is a filling of the Young diagram of $\lambda$ by positive integers, where the entries strictly increase along rows and weakly along columns. It is a standard Young tableau if there are no repeated entries.

\section{Maximum Length Chains in $\mathcal{T}_{n}{ }^{(m)}$ AND tableaux}

To each maximal chain $C$ in $\mathcal{T}_{n}{ }^{(m)}$, we associate a tableau $\Psi(C)$ of shape $(m(n-1), \ldots, m)$.

Definition 3.1. Let $C=\left\{\hat{1}=x_{r}>\ldots>x_{1}>x_{0}=\hat{0}\right\}$ be a maximal chain. As the chain is traversed from top to bottom, boxes are added. Fill the boxes added in moving from $x_{j}$ to $x_{j-1}$ with $r-j+1$. The resulting tableau is $\Psi(C)$. 
Every maximal chain is assigned a tableau and those of maximum length a standard Young tableaux. See Figure 2. $\Psi$ is injective. For the rest of this note, we focus on chains of maximum length.

$$
\begin{array}{|l|l|}
\hline 1 & 2 \\
\hline 3 &
\end{array}
$$

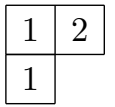

Figure 2. The tableau on the left corresponds to the chain on the left in Figure 1 and the tableau on the right to the chain on the right. The tableau on the left can be shifted by one, the one on the right cannot.

Definition 3.2. Let $m$ be a positive integer and $\lambda=\left(\lambda_{1}, \ldots, \lambda_{k}\right)$ be a partition such that $\lambda_{i}-\lambda_{i+1} \geq m$, for $i$ from 1 to $k-1$. Then the $m$-shifted diagram of $\lambda$ is obtained from the usual diagram of $\lambda$ by moving the $i^{\text {th }}$ row $m(i-1)$ boxes to the right, for $i>1$. An $m$-shifted tableau of shape $\lambda$ is a filling of the $m$-shifted diagram of $\lambda$ by positive integers such that the entries strictly increase along rows and weakly along columns. It is standard if no entry is repeated and the entries are from $\{1,2, \ldots,|\lambda|\}$.

The case $m=1$ is the usual shifted tableau.

Theorem 3.3. The number of chains of maximum length in the $m$-Tamari lattice $\mathcal{T}_{n}{ }^{(m)}$ is equal to the number of standard $m$-shifted tableaux of shape $(m(n-1), m(n-$ $2), \ldots, m)$.

Proof. $\mathcal{T}_{n}$ has a chain of length $\left(\begin{array}{l}n \\ 2\end{array}\right)$, and this is the maximum length [Mar92. In $\mathcal{T}_{n}{ }^{(m)}$, consider the chain whose vertex $x_{i}$ is the $(m, n)$-Dyck partition $\lambda=$ $\left(\lambda_{1}, \ldots, \lambda_{n}\right)$, where

$$
\lambda_{j}= \begin{cases}m(n-j) & \text { if } m\left(\begin{array}{l}
n \\
2
\end{array}\right)-i \geq \sum_{h=1}^{j} m(n-h), \\
m\left(\begin{array}{l}
n \\
2
\end{array}\right)-i-\sum_{h=1}^{j-1} m(n-h) & \text { otherwise. }\end{cases}
$$

This is a chain of length $m\left(\begin{array}{l}n \\ 2\end{array}\right)$ and there can be no longer ones.

Let $N$ denote $m\left(\begin{array}{c}n \\ 2\end{array}\right)$. A maximum length chain $C=\left\{\hat{0}=x_{0} \lessdot x_{1} \lessdot \ldots \lessdot\right.$ $\left.x_{N-1} \lessdot x_{N}=\hat{1}\right\}$ in $\mathcal{T}_{n}{ }^{(m)}$ is one where the Young diagram for $x_{j}$ has exactly one square more than the Young diagram for $x_{j+1}$, for $j$ from 0 to $N-1$. This is only possible if $k(\lambda, i)=i$ in Definition 2.2 whenever $x_{j}=\left(\lambda_{1}, \ldots, \lambda_{n}\right)$ and $x_{j+1}=\left(\lambda_{1}, \ldots, \lambda_{k(\lambda, i)-1}, \lambda_{k(\lambda, i)}-1, \ldots, \lambda_{i}-1, \lambda_{i+1}, \ldots, \lambda_{n}\right)$. Thus for each cover in the maximum length chain, when a box is removed from row $i$ of $x_{j}$, we have $i=1$ or $\lambda_{i-1}-\lambda_{i} \geq m$. To express this in terms of the entries of the tableau $\Psi(C)$, let $b_{g h}$ denote the entry in row $g$ and column $h$. Then the condition $i=1$ or $\lambda_{i-1}-\lambda_{i} \geq m$ becomes $i=1$ or $b_{i, h}>b_{i-1, h+m}$.

This is exactly the property that the entries in a tableau must have if we are to be able to shift that tableau by $m$.

Conversely, given such a tableau, the conditions on the entries guarantee that it represents a maximum length chain in $\mathcal{T}_{n}$. 
Corollary 3.4. The number of chains of length $\left(\begin{array}{l}n \\ 2\end{array}\right)$ in $\mathcal{T}_{n}$ is

$$
\left(\begin{array}{l}
n \\
2
\end{array}\right) ! \frac{(n-2) !(n-3) ! \cdots 1 !}{(2 n-3) !(2 n-5) ! \cdots 1 !} .
$$

Proof. Use the Thrall formula Thr52 for the number of shifted tableaux of shape $(n-1, n-2, \ldots, 1)$.

There are $2^{n-1}$ partitions with distinct parts whose Young diagram is contained within the Young diagram for $(n-1, \ldots, 1)$. These partitions label the vertices which appear in a chain of maximum length.

\section{Maximal chains in weak Bruhat order}

Let $\mathcal{S}_{n}$ be the symmetric group with simple transpositions $S$. Each $w$ in $\mathcal{S}_{n}$ can be written as a word in $S$, and any word for $w$ which is minimal in length among words for $w$ is called a reduced expression for $w$. The length of $w$ is the length of any reduced expression for $w$ and is denoted $\ell(w)$. To define the support of $w$, written $\operatorname{Sup}(w)$, let $s_{i_{1}} \ldots s_{i_{\ell w}}$ be any reduced word for $w$. Then $\operatorname{Sup}(w) \subseteq S$ is the set $\left\{s_{i_{1}}, s_{i_{2}}, \ldots, s_{i_{\ell(w)}}\right\}$. Any reduced expression for $w$ can be transformed into any other by a sequence of braid relation transformations, which means that $\operatorname{Sup}(w)$ is independent of the reduced expression for $w$. The (right) weak (Bruhat) order on $\mathcal{S}_{n}$ is the transitive closure of the cover relation $w \lessdot w s$ whenever $s \in S$ and $\ell(w)<\ell(w s)$. See Figure 3. The identity permutation is the minimum element $\hat{0}$, and the maximum element $\hat{1}$ is $w_{0}$, where $w_{0}(i)=n-i+1$. The symmetric group, together with the weak order, form a lattice, which we also denote by $\mathcal{S}_{n}$.

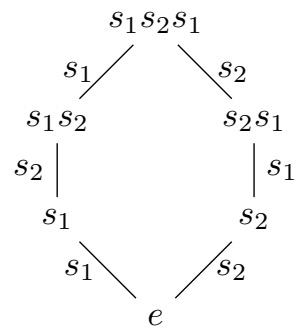

Figure 3 . The Hasse diagram of the poset $\mathcal{S}_{3}$. The edge from $w$ to $w s$ is labeled by $s$.

All maximal chains in $\mathcal{S}_{n}$ have the same length, $N=\left(\begin{array}{c}n \\ 2\end{array}\right)$. Stanley Sta84] conjectured and proved using symmetric functions that the number of maximal chains in $\mathcal{S}_{n}$ is the number of standard Young tableaux of shape $(n-1, n-2, \ldots, 1)$. Edelman and Greene [EG87] re-proved this result using a bijection, described here in Section 4.1. On the other hand, Björner and Wachs [BW97] and later Reading Rea06 showed that $\mathcal{T}_{n}$ can be considered as a sublattice of $\mathcal{S}_{n}$ and that there is an order preserving projection from $\mathcal{S}_{n}$ to $\mathcal{T}_{n}$. We describe Reading's approach in Section 4.2. In Section 4.3. Theorem 4.6, we describe how the Edelman-Greene bijection is related to the function $\Psi$ from Section 3 by the inclusion of $\mathcal{T}_{n}$ in $\mathcal{S}_{n}$. Note that the Stanley lattice refines $\mathcal{T}_{n}$ [BB09] and has the same number of maximal chains as $\mathcal{S}_{n}$. However, we are interested in comparing $\mathcal{T}_{n}$ to $\mathcal{S}_{n}$, instead of to the Stanley lattice, because of the algebraic structure inherent to $\mathcal{S}_{n}$. 
4.1. Maximal chains in $\mathcal{S}_{n}$ and standard Young tableaux. A maximal chain in $\mathcal{S}_{n}$ is of the form $\hat{0}=x_{0} \lessdot x_{1} \lessdot \ldots \lessdot x_{N-1} \lessdot x_{N}=\hat{1}$, where $x_{k-1} s_{i_{k}}=x_{k}$ and $\ell\left(x_{k-1}\right)+1=\ell\left(x_{k}\right)$, for $1 \leq k \leq N$. Each maximal chain can be seen as a reduced word $s_{i_{1}} \ldots s_{i_{N}}$ for $w_{0}$, and we will use both ways of indicating a chain.

EG87] defines a bijection between maximal chains in $\mathcal{S}_{n}$ and standard Young tableaux of shape $(n-1, \ldots, 1)$. The authors define an analog of the RobinsonSchensted-Knuth correspondence for reduced expressions. Here we assume the reader is familiar with the usual Robinson-Schensted-Knuth insertion and review the Coxeter-Knuth insertion from [EG87.

Definition 4.1 ([EG87], Definition 6.20, Coxeter-Knuth insertion). Suppose that $T$ is a tableau with rows $T_{1}, T_{2}, \ldots, T_{j}$ and $x_{0}$ is to be inserted into $T_{1}$. For each $i \geq 0$ add $x_{i}$ to row $T_{i+1}$, bumping (perhaps) $x_{i+1}$ to the next row, using the usual Robinson-Schensted-Knuth insertion, except in the following special case. If $x_{i}$ bumps $x_{i+1}$ from row $T_{i+1}, x_{i+1}=x_{i}+1$, and $x_{i}$ is already present in $T_{i+1}$, the value of $x_{i}$ in $T_{i+1}$ is changed from $x_{i}$ to $x_{i+1}$.

Also in their words, if $x$ is inserted into a row containing $x x+1$, a copy of $x+1$ is bumped to the next row, but the original $x x+1$ remains unchanged.

For the bijection: begin with a maximal chain in $\mathcal{S}_{n}$, written as a reduced expression $s_{i_{1}} \ldots s_{i_{N}}$ for $w_{0}$. Coxeter-Knuth insert its indices in reverse into the empty tableau to obtain a pair $(P, Q)$ of tableaux of shape $(n-1, n-2, \ldots, 1)$. The Edelman-Greene bijection matches the chain with the standard Young tableau $Q$. See Figure 4.

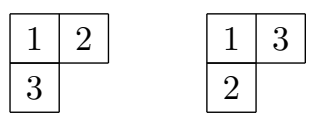

Figure 4 . The tableau on the left corresponds to the chain $s_{1} s_{2} s_{1}$ on the left in Figure 3. and the tableau on the right to the chain $s_{2} s_{1} s_{2}$ on the right. The tableau on the left can be shifted by one, the one on the right cannot.

4.2. $c$-sorting. Björner and Wachs BW97 show that $\mathcal{T}_{n}$ is induced by weak order on a certain set of permutations, 312-avoiding permutations, and is also a quotient of weak order. For the latter, they give a projection from $\mathcal{S}_{n}$ to $\mathcal{T}_{n}$. Reading [Rea06] introduced $c$-sorting words, which generalize the 312 -avoiding permutations. He used them to define Cambrian lattices and thereby generalized the Tamari lattice to any Weyl group $W$ and Coxeter word $w \in C$. He defined a projection which generalizes the Björner-Wachs projection. The Tamari lattice is the case $c=s_{1} s_{2} \ldots s_{n-1}$ Cambrian lattice. We use the Reading description of $\mathcal{T}_{n}$ as a sublattice of $\mathcal{S}_{n}$, although in some sense it is more general than we need, because it explicitly describes the Tamari lattice in terms of elements of $S$, the simple transpositions.

Throughout this note, $c$ will be the Coxeter word $s_{1} s_{2} \ldots s_{n-1}$. For a set $K=$ $\left\{a_{1}<a_{2}<\ldots<a_{r}\right\} \subset[n]$, let $c_{K}$ denote $s_{a_{1}} s_{a_{2}} \ldots s_{a_{r}}$. There may be many different ways to write $w \in \mathcal{S}_{n}$ as a reduced subword of $c^{\infty}=\operatorname{ccccc} \ldots$. The $c$ sorting word of $w \in \mathcal{S}_{n}$ is the reduced subword of $c^{\infty}$ for $w$ which is lexicographically 
first, as a sequence of positions in $c^{\infty}$. The $c$-sorting word for $w$ can be written as $c_{K_{1}} c_{K_{2}} \ldots c_{K_{p}}$, where $p$ is minimal for the property

$$
w=c_{K_{1}} c_{K_{2}} \ldots c_{K_{p}} \text { and } \quad \ell(w)=\sum_{i=1}^{p}\left|K_{i}\right| .
$$

An element $w \in \mathcal{S}_{n}$ with $c$-factorization $c_{K_{1}} c_{K_{2}} \ldots c_{K_{p}}$ is called c-sortable if $K_{p} \subset K_{p-1} \subset \ldots \subset K_{1}$.

Example 4.2. In $\mathcal{S}_{4}$, let $w=3241$ and $v=4132$. $w$ has the three reduced expressions: $s_{1} s_{2} s_{3} s_{1}, s_{1} s_{2} s_{1} s_{3}$, and $s_{2} s_{1} s_{2} s_{3}$. Its $c$-sorting word is $s_{1} s_{2} s_{3} s_{1}=$ $c_{\{1,2,3\}} c_{\{1\}}$ and it is $c$-sortable. $v$ also has three reduced expressions: $s_{3} s_{2} s_{3} s_{1}$, $s_{2} s_{3} s_{2} s_{1}$, and $s_{3} s_{2} s_{1} s_{3}$. Its $c$-sorting word is $s_{2} s_{3} s_{2} s_{1}=c_{\{2,3\}} c_{\{2\}} c_{\{1\}}$ and it is not $c$-sortable.

Rea06] defines the map $\pi_{\downarrow}^{c}$, which takes an element $w$ of $\mathcal{S}_{n}$ to the maximal $c$ sortable word below $w$. See Figure 5 . In the case $c=s_{1} s_{2} \ldots s_{n-1}$, this map is used to show that the Tamari lattice $\mathcal{T}_{n}$ is a lattice quotient of $\mathcal{S}_{n}$. Reading considers $\mathcal{T}_{n}$ as an induced sublattice of $\mathcal{S}_{n}$ and labels the elements of $\mathcal{T}_{n}$ by $c$-sortable words. Maximum length chains in $\mathcal{T}_{n}$ can be identified with certain maximal chains in $\mathcal{S}_{n}$.
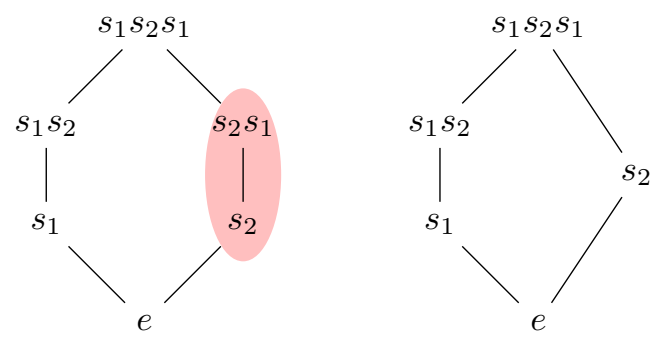

Figure 5. The Hasse diagrams of the posets $\mathcal{S}_{3}$ and $\mathcal{T}_{3}$, with vertices now labeled by $c$-sorting words. Under $\pi_{\downarrow}^{c}$, the vertices $s_{2}$ and $s_{2} s_{1}$ are both mapped to the same vertex in $\mathcal{T}_{3}$, the vertex labeled with $s_{2}$.

We will need the following lemma to characterize reduced expressions for $w_{0}$ in $\mathcal{S}_{n}$ coming from maximal length chains in $\mathcal{T}_{n}$. It is a very slightly modified version of a lemma from HLT11.

Lemma 4.3 (HLT11, Lemma 2.6). Suppose $w$ and $w s_{k}$ are both c-sortable and $\ell\left(w s_{k}\right)=\ell(w)+1$. Suppose $w$ 's c-factorization is $c_{K_{1}} \ldots c_{K_{p}}$. Then the c-factorization of $w s_{k}$ is either $c_{K_{1}} \ldots c_{K_{p}} c_{\{k\}}$ or $c_{K_{1}} \ldots c_{K_{i} \cup\{k\}} \ldots c_{K_{p}}$.

If $w s_{k}=c_{K_{1}} \ldots c_{K_{i} \cup\{k\}} \ldots c_{K_{p}}$, then $i$ is uniquely determined and $s_{k}$ commutes with every $s_{h}$ for $h \in K_{i+1} \cup M$, where $M=\left\{m \in K_{i} \mid m>k\right\}$.

Proof. We include, almost verbatim, the proof from [HLT11 for completeness.

If $k \in K_{p}$, the $c$-factorization of $w s_{k}$ is simply $c_{K_{1}} \ldots c_{K_{p}} c_{\{k\}}$, so assume $k \notin K_{p}$. Let $c_{L_{1}} \ldots c_{L_{q}}$ be the $c$-factorization of $w s_{k}$. Since $\ell\left(\left(w s_{k}\right) s_{k}\right)<\ell\left(w s_{k}\right)$, by the exchange property (see [BB05]) there is a unique $i, 1 \leq i \leq q$ and $r \in L_{i}$, such that $w=c_{L_{1}} \ldots c_{L_{i} \backslash\{r\}} \ldots c_{L_{q}}$. 
Case 1. Suppose $i=1$; that is, 1 is the unique index such that

$$
w=c_{L_{1} \backslash\{r\}} c_{L_{2}} \ldots c_{L_{q}} .
$$

First we show that $r$ is not a member of $K_{1}$.

Suppose, for contradiction, that $r \in K_{1}=\operatorname{Sup}(w)$. Since $c_{L_{1} \backslash\{r\}} c_{L_{2}} \ldots c_{L_{q}}$ is reduced and $L_{2} \supseteq \cdots \supseteq L_{q}$ is nested, we have $r \in L_{2}$. Hence

$$
K_{1}=\operatorname{Sup}(w)=\left(L_{1} \backslash\{r\}\right) \cup L_{2}=L_{1} \cup L_{2}=L_{1} .
$$

Thus $c_{L_{2}} \ldots c_{L_{q}}$ and $c_{K_{2}} \ldots c_{K_{q}} s_{k}$ are reduced expressions for some $\hat{w} \in W$ and $\ell\left(\hat{w} s_{k}\right)<\ell(\hat{w})$. The exchange condition implies the existence of a unique index $j$, $2<j<q$ and $t \in L_{j}$ such that

$$
\hat{w}=c_{L_{2}} \ldots c_{L_{j} \backslash\{t\}} \ldots c_{L_{q}} .
$$

In other words,

$$
w=c_{L_{1}} \hat{w}=c_{L_{1}} c_{L_{2}} \ldots c_{L_{j} \backslash\{t\}} \ldots c_{L_{q}}
$$

is reduced. But this contradicts the uniqueness of $i=1$ in (11). So $r \notin K_{1}$.

Since $r \notin K_{1}$, we know $r \notin \operatorname{Sup}(w)$ and $r \in \operatorname{Sup}\left(w s_{k}\right)$, which shows that $k=r$. Because $s_{k} \notin \operatorname{Sup}(w)$, in order to rewrite $s w_{k}$ in its $c$-sorting form, we must have commuted $s_{k}$ from the right to the left; i.e. $s_{k}$ commutes with $s_{h}$ for all $h \in K_{2} \cup M$.

Case 2. Suppose $i>1$. Then $K_{1}=\operatorname{Sup}(w)=L_{1}$. Set $\nu:=\min (p, i-1)$ and iterate the argument for $c_{L_{1}}{ }^{-1}, c_{L_{2}}{ }^{-1} c_{L_{1}}{ }^{-1} w, \ldots$ to conclude $L_{j}=K_{j}$ for $1 \leq j \leq \nu$. If $\nu=p$, then $i=q=p+1$ and $L_{i} \backslash\{r\}=\emptyset$. So $L_{i}=\{j\} \subseteq L_{i-1}=K_{p}$, which contradicts $k \notin K_{p}$. Thus $\nu=i-1$ for some $i \leq p$ and $L_{j}=K_{j}$ for $1 \leq j \leq i-1$. Thus, we may assume $i=1$, and we are done by Case 1 .

In a chain of maximum length in $\mathcal{T}_{n}$, the factorizations change by exactly one transposition as we move up the chain. Thus Lemma 4.3, combined with the fact that $s_{j}$ and $s_{j-1}$ do not commute, has the following corollary.

Corollary 4.4. Let $v$ be a vertex in a chain of maximum length in $\mathcal{T}_{n}$ and $c=$ $s_{1} s_{2} \ldots s_{n-1}$. Suppose that $v$ 's c-factorization is $c_{K_{1}} \ldots c_{K_{p}}$. Then if $j$ is a member of $K_{i}$, either $j-1 \in K_{i}$ or $j=1$. Additionally, if $j \leq n-i$ and $j \notin K_{i}$, then $j-1 \notin K_{i+1}$.

Let $\lambda$ be a partition. A lattice permutation of shape $\lambda$ is a sequence $a_{1} a_{2} \ldots a_{N}$ in which $i$ occurs $\lambda_{i}$ times and such that in any left factor $a_{1} \ldots a_{j}$, the number of $i$ 's is at least as great as the number of $i+1$ 's. See Sta99. We combine Lemma 4.3 with Corollary 4.4 to obtain Corollary 4.5 .

Corollary 4.5. Let $s_{i_{1}} \ldots s_{i_{N}}$ be a reduced word for $w_{0}$ coming from a maximum length chain in $\mathcal{T}_{n}$. Let $C=i_{1} i_{2} \ldots i_{N}$ and $C^{R}=i_{N} \ldots i_{1}$. Then both $C$ and $C^{R}$ are lattice permutations for the partition $\lambda=(n-1, n-2, \ldots, 1)$.

4.3. Maximal length chains in Tamari order and weak Bruhat order. Theorem 4.6 and the results from Section 4 explain that the modified RobinsonSchensted algorithm encodes the Tamari order when used on certain chains in the weak Bruhat order on $\mathcal{S}_{n}$.

Theorem 4.6. Let $C=\left\{\hat{0}=x_{0} \lessdot x_{1} \lessdot \ldots \lessdot x_{N-1} \lessdot x_{N}=\hat{1}\right\}$ be a maximum length chain in $\mathcal{T}_{n}$. Each $x_{i}$ is c-sortable, so it may also be considered as a chain in $\mathcal{S}_{n}$. As a chain in $\mathcal{T}_{n}, C$ maps to a standard Young tableau $T$ as in Section 3 , As a chain in $\mathcal{S}_{n}$, it maps to a standard Young tableau $T^{\prime}$ as in Section 4 . Then we have $T=T^{\prime}$. 
Proof. First we will define a bijection $p$ between $c$-sortable words and $(m, n)$-Dyck partitions for $m=1$ and show that it respects the covering relation in $\mathcal{T}_{n}$. We then show that if $w \lessdot w s_{i_{k}}$, then $p\left(w s_{i_{k}}\right)$ is the partition $p(w)$ with column $i_{k}$ shortened by 1 . Lastly, we show that if we insert $i_{k}$ into the tableau produced by $s_{i_{N}} \ldots s_{i_{k+1}}$, we lengthen column $i_{k}$ by 1 . See Figure 6 .

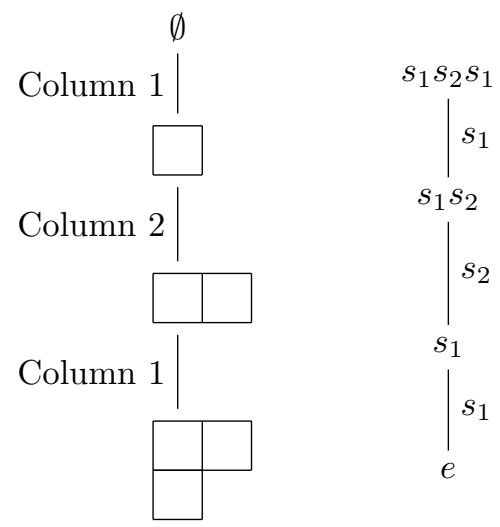

Figure 6 . On the left is the single maximum length chain in $\mathcal{T}_{3}$, and on the right is the maximum length chain in $\mathcal{S}_{3}$ that it corresponds to under $p$. If 121 is inserted into $\emptyset$ using Coxeter-Knuth insertion, the sequence of shapes we obtain is the same as those in the chain on the left.

Given a $c$-sortable word $w$, with $c$-factorization $c_{K_{1}} \ldots c_{K_{p}}$, let $p(w)$ be the partition where column $i$ has length $n-i-\#$ of occurrences of $i$ in $w$ 's factorization. See also [BK01, Kra01, Ful03. The partition $p(w)$ can also be described as follows. Let $X$ be the partition $(n-1, n-2, \ldots, 1)$, the minimal element of $\mathcal{T}_{n}$. The $k^{\text {th }}$ diagonal of $X$ is the set of boxes $(i, j)$ such that $i+j-1=k$. Label the boxes in column $i$ by $i$. Then $p(w)$ is the partition $X$ with the box labeled $i$ in diagonal $k$ removed if and only if $i \in K_{k}$. Then since $K_{p} \subset K_{p-1} \subset \ldots \subset K_{1}, p(w)$ is a partition.

If $w \lessdot w s_{j}$, then by Lemma 4.3 and the definition of $p$, we have that $p\left(w s_{j}\right)$ is the partition $p(w)$ with column $j$ shortened by 1 . Write $p(w)=\left(a_{1}, \ldots, a_{n}\right)$ and $p\left(w s_{j}\right)=\left(a_{1}, \ldots, a_{i-1}, a_{i}-1, a_{i+1}, \ldots, a_{n}\right)$, where $a_{i}=j$. The box removed from the diagram of $p(w)$ was on diagonal $i+j-1$. Since this box was present if $p(w)$, we know that $j \notin c_{K_{i+j-1}}$ in the $c$-factorization of $w$. Thus by Corollary 4.4 $j+1 \notin c_{K_{i+j-1}}$, so that $a_{i-1}>a_{i}$ and $p(w) \lessdot p\left(w s_{j}\right)$ according to Definition 2.2.

Let $C=s_{i_{1}} \ldots s_{i_{N}}$ be a maximal chain in $\mathcal{S}_{n}$ which is also a maximal length chain in $\mathcal{T}_{n}$. Suppose $s_{i_{N}}, \ldots, s_{i_{k+1}}$ have been inserted as in Section 4 to form a tableau $P$ of shape $\lambda$. For ease of notation, write $j$ for $s_{j}$. Let $x, y$, and $z$ be the number of occurrences of $i_{k}-1, i_{k}$, and $i_{k}+1$, respectively, in $s_{i_{N}} \ldots s_{i_{k-1}}$. Since both $C$ and $C^{R}$ are lattice words and $j$ appears a total of $N-j$ times in $C$, we have that $x=y+1$ and $y=z$. Thus, by induction, column $i_{k}-1$ has length $x$ and both column $i_{k}$ and $i_{k}+1$ have length $x-1$. In row $h$ of $P$, we have $i_{k}+h-2$, $i_{k}+h-1$, and $i_{k}+h$ in columns $i_{k}-1, i_{k}$, and $i_{k}+1$ respectively. Again, by 
induction, when $i_{k}+h-1$ is inserted into row $h$, for $h<x, i_{k}+h$ will be bumped into row $h+1$. Finally, at row $x, i_{k}+x-1$ will settle in column $i_{k}$, finishing the proof.

\section{REFERENCES}

[BB05] Anders Björner and Francesco Brenti, Combinatorics of Coxeter groups, Graduate Texts in Mathematics, vol. 231, Springer, New York, 2005. MR2133266 (2006d:05001)

[BB09] Olivier Bernardi and Nicolas Bonichon, Intervals in Catalan lattices and realizers of triangulations, J. Combin. Theory Ser. A 116 (2009), no. 1, 55-75, DOI 10.1016/j.jcta.2008.05.005. MR.2469248 (2009m:05004)

[BFP11] Mireille Bousquet-Mélou, Éric Fusy, and Louis-François Préville-Ratelle, The number of intervals in the m-Tamari lattices, Electron. J. Combin. 18 (2011), no. 2, Paper 31, 26 pp. MR.2880681

[BK01] Jason Bandlow and Kendra Killpatrick, An area-to-inv bijection between Dyck paths and 312-avoiding permutations, Electron. J. Combin. 8 (2001), no. 1, Research Paper 40, 16 pp. (electronic). MR:1877659 (2003c:05009)

[BP11] F. Bergeron and L. F. Préville-Ratelle, Higher trivariate diagonal harmonics via generalized Tamari posets, J. Comb. 3 (2012), no. 3, 317-341. MR3029440

[BW97] Anders Björner and Michelle L. Wachs, Shellable nonpure complexes and posets. II, Trans. Amer. Math. Soc. 349 (1997), no. 10, 3945-3975, DOI 10.1090/S0002-9947-9701838-2. MR 1401765 (98b:06008)

[DM47] A. Dvoretzky and Th. Motzkin, A problem of arrangements, Duke Math. J. 14 (1947), 305-313. MR0021531 (9,75i)

[EG87] Paul Edelman and Curtis Greene, Balanced tableaux, Adv. in Math. 63 (1987), no. 1, 42-99, DOI 10.1016/0001-8708(87)90063-6. MR871081 (88b:05012)

[FT67] Haya Friedman and Dov Tamari, Problèmes d'associativité: Une structure de treillis finis induite par une loi demi-associative (French), J. Combinatorial Theory 2 (1967), 215-242. MR 0238984 (39 \#344)

[Ful03] Markus Fulmek, Enumeration of permutations containing a prescribed number of occurrences of a pattern of length three, Adv. in Appl. Math. 30 (2003), no. 4, 607-632, DOI 10.1016/S0196-8858(02)00501-8. MR1977846 (2004c:05009)

[Hai92] Mark D. Haiman, Dual equivalence with applications, including a conjecture of Proctor, Discrete Math. 99 (1992), no. 1-3, 79-113, DOI 10.1016/0012-365X(92)90368-P. MR1158783 (93h:05173)

[HLT11] Christophe Hohlweg, Carsten E. M. C. Lange, and Hugh Thomas, Permutahedra and generalized associahedra, Adv. Math. 226 (2011), no. 1, 608-640, DOI 10.1016/j.aim.2010.07.005. MR.2735770 (2012d:20085)

[HT72] Samuel Huang and Dov Tamari, Problems of associativity: A simple proof for the lattice property of systems ordered by a semi-associative law, J. Combinatorial Theory Ser. A 13 (1972), 7-13. MR0306064 (46 \#5191)

[Knu73] Donald E. Knuth, The art of computer programming. Volume 3, Sorting and searching. Addison-Wesley Series in Computer Science and Information Processing, Addison-Wesley Publishing Co., Reading, Mass.-London-Don Mills, Ont., 1973. MR0445948 (56 \#4281)

[Kra01] C. Krattenthaler, Permutations with restricted patterns and Dyck paths, Special issue in honor of Dominique Foata's 65th birthday (Philadelphia, PA, 2000), Adv. in Appl. Math. 27 (2001), no. 2-3, 510-530, DOI 10.1006/aama.2001.0747. MR1868978 (2002k:05005)

[Mar92] George Markowsky, Primes, irreducibles and extremal lattices, Order 9 (1992), no. 3, 265-290, DOI 10.1007/BF00383950. MR 1211380 (94a:06015)

[Rea06] Nathan Reading, Cambrian lattices, Adv. Math. 205 (2006), no. 2, 313-353, DOI 10.1016/j.aim.2005.07.010. MR 2258260 (2007g:05195)

[Sta84] Richard P. Stanley, On the number of reduced decompositions of elements of Coxeter groups, European J. Combin. 5 (1984), no. 4, 359-372. MR782057 (86i:05011)

[Sta99] Richard P. Stanley, Enumerative combinatorics. Vol. 2, with a foreword by Gian-Carlo Rota and appendix 1 by Sergey Fomin. Cambridge Studies in Advanced Mathematics, 62, Cambridge University Press, Cambridge, 1999. MR1676282 
[Tam62] Dov Tamari, The algebra of bracketings and their enumeration, Nieuw Arch. Wisk. (3) 10 (1962), 131-146. MR0146227 (26 \#3749)

[Thr52] R. M. Thrall, A combinatorial problem, Michigan Math. J. 1 (1952), 81-88. MR0049844 $(14,234 \mathrm{~g})$

School of Mathematical and Statistical Sciences, Arizona State University, Tempe, Arizona 85287

School of Mathematical and Statistical Sciences, Arizona State University, Tempe, ARIZONA 85287 